\title{
A new piecewise linear Chen system of fractional-order; Numerical approximation of stable attractors
}

\author{
Marius-F. Danca* \\ Department of Mathematics and Computer Science, Avram Iancu University, \\ 400380 Cluj-Napoca, Romania, \\ and \\ Romanian Institute of Science and Technology, \\ 400487 Cluj-Napoca, Romania \\ Email: danca@rist.ro \\ M.A. Aziz-Alaoui \\ LMAH, FR-CNRS-3335, ULH, ISCN, Normandie University, \\ BP 540, 76058, Le Havre Cedex, France \\ Email: aziz.alaoui@univ-lehavre.fr \\ Michael Small \\ School of Mathematics and Statistics (M019), University of Western Australia, \\ Crawley, WA 6009, Australia \\ Email: michael.small@uwa.edu.au
}

December 30, 2014

Dedicated to Professor Guanrong(Ron) Chen on the occasion of his 65th birthday

\section{Abstract}

In this paper we present a new version of Chen's system - a piecewise linear Chen system of fractional-order. Via a sigmoid-like function, the discontinuous system is transformed into a continuous system. By numerical simulations, we reveal chaotic behaviors and also mulistability, i.e. the existence of small parameter windows where, for some fixed bifurcation parameter and depending on initial conditions, coexistence of stable attractors and chaotic attractors is possible. Moreover, we show that by using an algorithm to switch the bifurcation parameter, the stable attractors can be numerically approximated.

Keywords: PWL Chen attractor of fractional-order; parameter switching; Cellina's Theorem, Filippov regularization; Sigmoid function; Bifurcation diagram

PACS 05.45.Ac, 05.45.Gg, 05.45.Pq

\section{Introduction}

There are several paradigmatic three dimensional chaotic flows. Aside from the ubiquitous Lorenz and Rössler systems, one of the most intricate and widely studied is Chen's system, proposed in 1999 [1]. Each of these systems represent a topological distinct genus of chaos. In 2002 AzizAlaoui and Chen [2] presented a piecewise linear Chen system modeled by the following Initial Value Problem (IVP)

$$
\begin{aligned}
& \dot{x}_{1}=a\left(x_{2}-x_{1}\right), \\
& \dot{x}_{2}=\left(c-a-x_{3}\right) \operatorname{sgn}\left(x_{1}\right)+c d x_{2}, \quad x(0)=x_{0}, \quad t \in[0, T], \\
& \dot{x}_{3}=x_{1} \operatorname{sgn}\left(x_{2}\right)-b x_{3},
\end{aligned}
$$

\footnotetext{
${ }^{*}$ Corresponding author
} 
with $x_{0} \in \mathbb{R}^{3}, T>0, a, b, c, d$ some positive real parameters verifying the condition $a<c$.

Whereas the genuine Chen system [1] is a continuous system, having the following mathematical model

$$
\begin{aligned}
& \dot{x}_{1}=a\left(x_{2}-x_{1}\right), \\
& \dot{x}_{2}=(c-a) x_{1}-x_{1} x_{3}+c x_{2}, \quad x(0)=x_{0} \quad t \in[0, T], \\
& \dot{x}_{3}=x_{1} x_{2}-b x_{3},
\end{aligned}
$$

(with $a=35, b=3$, and $c=28$ ), the system (1), is a Piecewise Linear (PWL) system. The detailed asymptotic analysis of this system, including a study of the chaotic behavior with the bifurcation parameter, is presented in [2].

Even the fractional calculus is a topic of more than 300 years, recently, the systems of fractionalorder have attracted much attention after the first numerical methods have been performed. Fractional-order systems play an important role in many fields of science and engineering (see e.g. $[3,4,5,6,7,8,9,10,11]$ or $[12,13]$ with therein references). Also, piecewise continuous functions are used in the study of nonlinear circuits and systems. They are also commonly employed in the modeling of memristor, leading to many novel studies and applications (see e.g. $[14,15,16]$ ).

Entitled by the huge interest on Chen's system of integer and fractional-order (see e.g. [1] and [17]), we present in this paper a new and more general extension of the PWL system (1): the PWL Chen system of fractional-order, modeled by the following differential equations

$$
\begin{aligned}
& D_{*}^{q_{1}} x_{1}=a\left(x_{2}-x_{1}\right), \\
& D_{*}^{q_{2}} x_{2}=\left(c-a-x_{3}\right) \operatorname{sgn}\left(x_{1}\right)+c d x_{2}, \quad x(0)=x_{0}, \quad t \in[0, T], \\
& D_{*}^{q_{3}} x_{3}=x_{1} \operatorname{sgn}\left(x_{2}\right)-b x_{3},
\end{aligned}
$$

with $a=1.15, b=0.15, c=2$ and $d=0.1$.

$D_{*}^{q}$ (for $\left.q=\left(q_{1}, q_{2}, q_{3}\right)\right)$ stands for Caputo's differential operator of order $q$ with starting point 0 (see e.g. $[18,19,20]$ ). Recall that the Caputo differential operator is a fractional extension of differentiation defined for $n-1<q \leq n, q \in(0, \infty) \backslash \mathbb{N}, n$ being some positive integer, defined as follows

$$
D_{*}^{q} f(t)=\frac{1}{\Gamma(n-q)} \int_{0}^{t} \frac{f^{(n)}(\tau)}{(t-\tau)^{q+1-n}} d \tau
$$

where $\Gamma$ is Euler's function.

In contrast to alternative definitions of fractional differentiation, Caputo's derivative gives a physical interpretation to the included initial conditions, necessary in practical problems [21, 22]. Using this definition, one avoid the expression of initial conditions with fractional derivatives and therefore the initial condition can be considered in the standard form $x(0)=x_{0}$.

As in the most practical examples, in this paper we consider $q_{i} \leq 1, i=1,2,3$.

The chaotic behavior of PWL Chen's system (3) as function of the commensurate order $q$ is revealed by the Bifurcation Diagram (BD) in Fig.1(a) ${ }^{1}$. Few representative attractors are plotted in Fig.1(b)-(e)). As known, for systems of commensurate order, chaos persists over a minimal commensurate order $q_{\min }<1$, i.e. a system order $3 \times q_{\min }<3$. Revealed numerically by the BD, $q_{\min }$ for our system is about 0.865 , which indicate that chaos exists for system order greater than $3 \times q_{\min }=2.575$ (compare with the continuous Chen's system of fractional-order [12, 17]).

Let us denote by $p$ the single scalar bifurcation parameter, which for the considered system (3) can be any of the four parameters $a, b, c$ or $d$. Two representative cases have been considered here: $p:=d$, integer case $q=(1,1,1)$, and $p:=a$, integer case $q=(1,1,1)$, and the incommensurate fractional-order case $q=(0.99,0.98,0.97)$. For the sake of conciseness we do not present results of computation here, but we have found that the other two cases $(p:=b$ and $p:=c)$ demonstrate a similar behavior. We find particularly rich bifurcation behaviors, revealed by the BDs in Fig.2. The results are for: (a) $p:=d$ in the integer-order case, with $a=1.15$; (b) $p:=a$ in the integer order case; and (c) for $p:=a$ with the fractional-order case.

It is easy to verify that, in addition to the origin, the system has two equilibrium points $X^{*}\left( \pm \frac{a b-b c}{b c d \pm 1}, \pm \frac{a b-b c}{b c d \pm 1}, \pm \frac{a-c}{b c d \pm 1}\right)$ (Table 1). Because the equilibrium points reside in the plan $x_{1}=x_{2}$, $X_{2}^{*}$ and $X_{3}^{*}$ are not equilibrium points for this system (see [1]). The coordinates present symmetries

\footnotetext{
${ }^{1} \mathrm{BDs}$ are determined for positive values of the state variables.
} 
which are also evident in the simulations of the state variables $x_{1}$ and $x_{2}$. These symmetries come from the invariance of the vector field under rotation symmetry $(x, y, z) \rightarrow(-x,-y, z)$. To note that for the chosen values for parameters $a, b, c$ and $d$, with $a<c$, the state coordinate $x_{3}^{*}$ is positive while $x_{1,2}^{*}$ can be either positive and negative.

\begin{tabular}{c|c|c}
$\operatorname{sgn}\left(x_{1}\right)$ & $\operatorname{sgn}\left(x_{2}\right)$ & $X^{*}\left(x_{1}^{*}, x_{2}^{*}, x_{3}^{*}\right)$ \\
\hline- & - & $X_{1}^{*}\left(-\frac{b a-b c}{b c d-1},-\frac{b a-b c}{b c d-1}, \frac{a-c}{b c d-1}\right)$ \\
\hline+ & - & $X_{2}^{*}\left(\frac{b a-b c}{b c d+1}, \frac{b a-b c}{b c d+1},-\frac{a-c}{b c d+1}\right)$ \\
\hline- & + & $X_{3}^{*}\left(-\frac{b a-b c}{b c d+1},-\frac{b a-b c}{b c d+1},-\frac{a-c}{b c d+1}\right)$ \\
\hline+ & + & $X_{4}^{*}\left(\frac{b a-b c}{b c d-1}, \frac{b a-b c}{b c d-1}, \frac{a-c}{b c d-1}\right)$
\end{tabular}

Table 1: Equilibrium points of the PWL Chen system (3).

As can be seen from the detail in Figure $2(\mathrm{a})$ and (b), for $p:=d$ and $p:=a$ respectively, a new characteristic has been uncovered for this system, the multistability, i.e. the coexistence of regular and chaotic motions in some parameter windows (details $D_{1}$ in Figure 2 (a) and $D_{2}$ in Figure 2 (b) ). Therefore, for a specific value $p$ in these windows, depending on the basin of attraction and initial conditions, we can find coexisting attractors. For example, for $a=1.15, b=0.15, c=0.1$, and with $p:=d=0.361$ chosen in the window $[0.345,0.370]$, we found two different attractors: a chaotic one and a stable cycle (see $D_{1}$ in Fig.2(a)). For $b=0.15, c=2, d:=0.1$ and with $p:=a$ chosen in the window $p \in[0.7,0.8]$, the two attractors are plotted in the detail $D_{2}$ in Fig.2(b).

In the remainder of this paper we show numerically, aided by computer simulations, that every stable attractor of Chen's system (3) can be numerically approximated by a Parameter Switching (PS) algorithm [23]. The PS algorithm switches $p$ within a chosen set, while the IVP is numerically integrated with some numerical scheme with fixed step size. The convergence of the PS algorithm to some desired attractor is presented in [24, 25].

The paper is structured as follows: in Section 2, the PS algorithm is explained, Section 3 presents the continuous approximation of the IVP (3) and in Section 4 several stable attractors of the PWL Chen system of fractional-order are approximated with the PS algorithm.

\section{Parameter switching algorithm}

Let a continuous system of fractional-order be modeled by the following IVP

$$
D_{*}^{q} x=f(x)+p A x, \quad t \in I=[0, T], \quad x(0)=x_{0},
$$

where: $A \in \mathbb{R}^{n \times n}$ is a real square constant matrix; $f: \mathbb{R}^{n} \rightarrow \mathbb{R}^{n}$ is a nonlinear, at least continuous function and $q \leq 1$. The great majority of known systems of integer or fractional-order, including systems like: Lorenz, Chen, Rössler, Chua, Rikitake and many others systems, are modeled by the IVP (4). In $[24,25]$ it was proved analytically and verified numerically that the PS algorithm allows the numerical approximation of any desired attractor, by switching the control parameter within a chosen finite set of values while the underlying IVP is numerically integrated with some fixed step size numerical method.

Next, assume that the IVP enjoys the uniqueness Lipschitz continuity which is a common sufficient condition for both integer and fractional case (see [26] Corollary 6.9 for FDE uniqueness). Furthermore, denote by $\mathcal{P}_{N}=\left\{p_{1}, p_{2}, \ldots, p_{N}\right\} \subset \mathbb{R}, \quad N \geq 2$, the switching values set.

Remark 1. Due to uniqueness assumption, it is reasonable to consider that to each $p_{i} \in \mathcal{P}_{N}$, $i \in\{1,2, \ldots, n\}$, it corresponds a unique attractor $A_{p_{i}}$. As usual for numerical tests, in this paper we consider the attractors as being the trajectory of the underlying numerical solution, after sufficiently long transients are removed.

By choosing $\mathcal{P}_{N}$, while the underlying IVP is numerically integrated with a fixed step-size $h$, PS switches in some deterministic (periodic) way the control parameter within $\mathcal{P}_{N}$, for relative short time subintervals. The obtained "switched solution" will approximate the "averaged solution" 
obtained for $p$ replaced with the average of switched values. Following Remark 1, the attractor corresponding to the switched solution, will approximate as closely as desired (depending on the numerical integration accuracy limitation), the attractor corresponding to the averaged solution. The numerical integrations have been realized with the standard Runge-Kutta (RK4) method for the integer-order case and Adams-Bashforth-Moulton (ABM) method [22] for the fractional-order case (see also [27]).

Schematically, for a chosen set $\mathcal{P}_{N}$ with the "weights" set $\mathcal{M}=\left\{m_{1}, m_{2}, \ldots, m_{N}\right\}$, and a fixed step-size $h$, the way in which PS algorithm works can be expressed schematically as follows

$$
\left[m_{1} p_{1}, m_{2} p_{2}, \ldots, m_{N} p_{N}\right] \text {. }
$$

The scheme (5) means that for the first $m_{1}$ integration steps the control parameter $p$ will take the value $p_{1}$, then, for the next $m_{2}$ integration steps, $p=p_{2}$, and so on until, for $m_{N}$ times, $p=p_{N}$, after which, again $p=p_{1}$ for $m_{1}$ times, then $p=p_{2}$ for $m_{2}$ times and so on until the considered time integration interval, $[0, T]$, is covered. For example $\left[2 p_{1}, p_{2}\right]$ means that while the IVP is integrated, $p$ will take the values: $p=p_{1}, p=p_{1}, p=p_{2}, p=p_{1}, p=p_{1}, p=p_{2}$, and so on.

Let us denote the "weighted average" of the values of $\mathcal{P}_{N}$ by

$$
p^{*}:=\frac{\sum_{i=1}^{N} p_{i} m_{i}}{\sum_{i=1}^{N} m_{i}} .
$$

Then, the "switched attractor", $A^{*}$, obtained with the PS algorithm, will approximate the "averaged attractor", $A_{p^{*}}$, obtained by integration of the underlying IVP with $p$ replaced with $p^{*}$. The analytical proof of the convergence, for the integer-order case, is presented in [24, 25], while for fractional-order systems the convergence has been computationally verified for several systems (see e.g. [23]).

Remark 2. The PS algorithm can be used both as a control and an anticontrol-like techniques when, by some objective reasons, some certain targeted parameter value $p^{*}$ cannot be accessed directly. In this case, we have to select the set $\mathcal{P}_{N}, m_{i}, i=1,2, \ldots, N$ and an adequate scheme (5) to obtain, with (6), the targeted value $p^{*}$. For example, if the values of $\mathcal{P}_{N}$ generate chaotic behavior and $p^{*}$ generates a regular motion, the PS algorithm acts like a control-like algorithm. However, while most known control/anticontrol algorithms "force" the trajectory to change its characteristics and behavior, the PS algorithm allows to obtain approximation of any desired existing attractor.

Also, the PS algorithm can help to enrich our understanding of what happens in some real systems when the control parameter is switched by natural causes.

Finally, the PS algorithm proves that $p$ switchings present a robustness-like property in the following sense: for whatever sets $\mathcal{P}_{N}, \mathcal{M}$, if $p_{\text {min }}=\min \left\{\mathcal{P}_{N}\right\}$ and $p_{\text {max }}=\max \left\{\mathcal{P}_{N}\right\}$, the obtained values $p^{*}$ remains between $p_{\min }$ and $p_{\max }$. Note that the PS algorithm applies also for discrete-time real systems and some interesting results have been obtained for complex discrete-time systems [25], although that is beyond what we consider in this paper.

\section{Continuous approximation of PWL Chen's system}

The main obstacle in applying the PS algorithm to PWL systems is the lack of numerical methods specifically devised for fractional differential equations with discontinuous right-hand side (Filippov-like systems of fractional-order). This is one of the reasons that discontinuous systems of fractional-order have been not rigorously studied. One possible approach to surpass the obstacle of integration of discontinuous differential equations of fractional-order, is to approximate continuously the underlying discontinuous IVP, based on the transformation of the IVP into a set-valued one, which next can be continuously approximated with a single-value IVP (see [28]). Thus, piecewise constant components, like sgn, can be continuously approximated globally (in small neighborhoods of the graph) or locally (in small neighborhood centered at the discontinuity point $x=0$ ). In this paper we use the global sigmoid approximation $\widetilde{s g n}$

$$
\widetilde{\operatorname{sgn}}(x)=\frac{2}{1+e^{-\frac{x}{\delta}}}-1,
$$


which approximates globally the sgn function, on small neighborhoods of its graph. In Fig.3(a), for clarity, $\delta$ has be chosen larger $(\delta=1 e-1)$, while in Fig.3(b), sgn is drawn as a function of two variables: $x$ and $\delta$. The parameter $\delta$ controls the slope of the sigmoid function, near the discontinuity $x=0$. As proved in [28], for numerical purposes an adequate choice is $\delta=1 e-5$.

Using the sigmoid approximation (7), Chen's system (3) becomes

$$
\begin{aligned}
& D_{*}^{q_{1}} x_{1}=a\left(x_{2}-x_{1}\right), \widetilde{s g n}\left(x_{1}\right)+c d x_{2}, \\
& D_{*}^{q_{2}} x_{2}=\left(c-a-x_{3}\right) \\
& D_{*}^{q_{3}} x_{3}=x_{1} \widetilde{\operatorname{sgn}}\left(x_{2}\right)-b x_{3} .
\end{aligned}
$$

The cases studied in this paper are presented in Table 2.

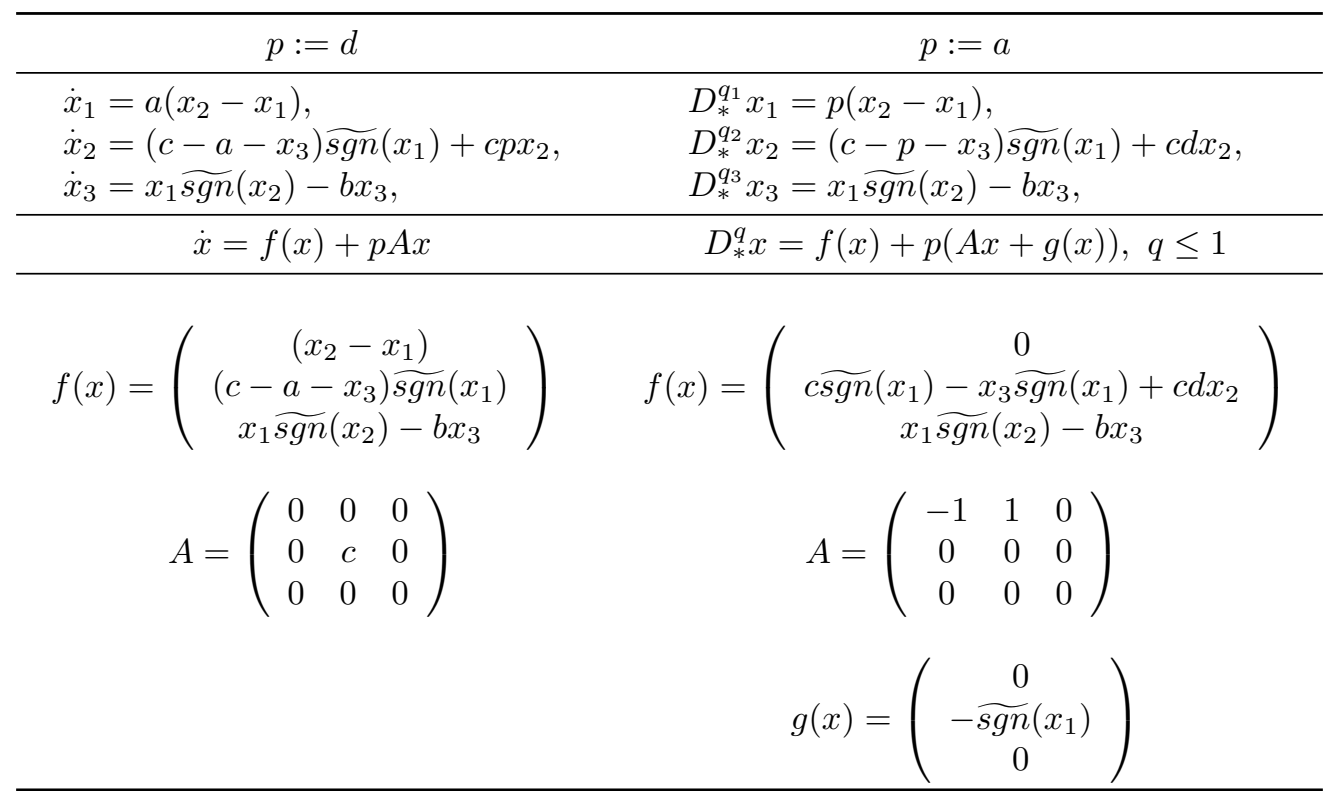

Table 2: PWL Chen systems analyzed in this paper.

Even for $p:=a$, the system does not belong to the class of systems modeled by the IVP (4), the PS algorithm still applies to this more general class of systems modeled by the following IVP (second column in Table 2 )

$$
D_{*}^{q} x=f(x)+p(A x+g(x)), \quad t \in I=[0, T], \quad x(0)=x_{0},
$$

where $g(x)=(0,-\widetilde{s g n}, 0)^{T}$.

\section{Numerical results}

As known, in contrast with integer-order derivatives, if a function of class $C^{n}$ is periodic, its derivative in the sense of Caputo, Riemann-Liouville and Grunwald- Letnikov, cannot be periodic with the same period (see e.g. [29]). However, in this paper we deal with numerical approaches of the continuous approximation of the PWL Chen system.

Next, we apply the PS algorithm to approximate stable cycles of Chen's PWL system of fractional-order (8), after it has been continuously approximated, for $p:=d$ integer-order case, and $p:=a$ integer and fractional-order cases. The case $p:=a$ is an example of a new class of systems of integer and fractional-order where the PS algorithm still applies. For this purpose, we chose the sets $\mathcal{P}$ and $\mathcal{M}$ which generate the targeted values $p^{*}$, after which the PS algorithm applies via the scheme (5). The targeted values for $p^{*}$ are chosen in the stable windows of BDs plotted in Fig.2. For the phase plots, the transients have been omitted. To underline numerically and 
computationally the match between the two attractors, $A^{*}$ and $A_{p^{*}}$, overplotted phase plots and time series have been used. The Hasudorff dimension, $d_{H}$ ([30] p. 114), is for all considered cases, of order of $1 e-3$, which indicates a good match between the two trajectories. In order to highlight the behavior characteristics of the utilized attractors, each illustrative case is accompanied by the underlying $\mathrm{BD}$, such that the chaotic or regular motion character can be easily deduced.

All the computations were made in Matlab, using the RK4 and ABM methods for the integerorder and fractional-order systems respectively, with the integration step-size of order of $1 e-3$.

- $p:=d$,

Commensurate case $q=(1,1,1)$

With $a=1.15, c=2$ and $b=0.15$, the system behaves chaotically (see BD in Fig.2(a)). Let us choose $\mathcal{P}_{2}=\{0.32,0.48\}$ (Fig.4(a)) and $\mathcal{M}=\{1,1\}$ with the underlying scheme, $\left[1 p_{1}, 1 p_{2}\right]$. The switched attractor $A^{*}$ approximates the averaged attractor $A_{p^{*}}$ with $p^{*}$ given by $(6): p^{*}=0.4$. The match between both attractors can be seen in the overplotted phase plots in Fig.4(b) and overplotted time series in Fig.4(e)-(g). The underlying attractors $A_{0.32}$ and $A_{0.48}$ are chaotic and regular motion respectively (Fig.4(c) and 4(d)).

- $p:=a$

Since the case $p:=d$ represents the case of the class of systems modeled by (4), where the PS algorithm has been extensively studied in previous works, we consider next the case $p:=a$, which belongs to the class of systems modeled by (9), and where the PS algorithm has been not applied yet. For $b=0.15, c=2$ and $d=0.1$, as can be seen from the BD in Fig.2(b), system dynamics are more complex than for $p:=d$, presenting several direct and reverse period-doubling bifurcations. In this case, due to the term $\widetilde{\operatorname{sgn}}\left(x_{1}\right)$, the system is modeled by (9). However, we will show that the PS algorithm still applies for this more general case.

Commensurate case $q=(1,1,1)$

Choosing $\mathcal{P}_{2}=\{0.9,1\}$, (see BD in Fig.5(a)), with $\mathcal{M}=\{1,1\}$, the relation (6) gives $p^{*}=0.95$, and with the scheme $\left[1 p_{1}, 1 p_{2}\right]$ the PS algorithm generates the switched attractor $A^{*}$, which approximates the stable cycle $A_{p^{*}}$ (see the phase plot in Fig.5(b) and time series in Fig.5(e)-(g)). Both underlying attractors $A_{1.9}$ and $A_{0.9}$ (Fig.5(c) and 5(d)) are chaotic. Therefore, in this case, the PS algorithm acts as a control-like method (see Remark 2).

The non-uniqueness of solutions for $p^{*}$ in the relation (6), allows different ways to obtain some desired attractor $A_{p^{*}}$. For example, the same attractor $A_{0.95}$ obtained above with the scheme $\left[1 p_{1}, 1 p_{2}\right]$ (with values $p_{1}=0.9$ and $p_{2}=1$, situated in closed neighborhoods of $p^{*}$ ), can be approximated by a switched attractor $A^{*}$ obtained with $p$ values situated relatively distant from $p^{*}$. For example, $A_{0.95}$ can be approximated with $P_{N}=\{0.67,1.51\}$ (Fig.6(a)) and weights $\mathcal{M}=\{2,1\}$, i.e. the scheme $\left[2 p_{1}, 1 p_{2}\right]$. Phase plots (Fig.6(b)) and time series (Fig.7(e)-(g)) reveal the approximation. This time, the stable cycle $A^{*}$ is obtained starting from parameter values which generate stable cycles (Fig.6(c) and 6(d)).

Also due to this same non-uniqueness, a set value $p^{*}$ can be obtained with $N>2$ elements. For example $p^{*}=0.95$ can be obtained with $\mathcal{P}_{6}=\{0.6,0.7,0.8,1.09,1.32,1.4\}$ (see Fig.7(a)) and $\mathcal{M}=\{1,2,2,3,1,1\}$. By applying the PS algorithm with the underlying scheme $\left[1 p_{1}, 2 p_{2}, 2 p_{3}, 3 p_{4}, 1 p_{5}, 1 p_{6}\right]$, one obtains again a good match between the two attractors $A^{*}$ and $A_{p^{*}}$ (Fig.7(b)-(k)).

Incommensurate case $q=(0.99,0.98,0.97)$

With $\mathcal{P}_{4}=\{0.75,1.29,1.52,1.6,1.38\}$ (see BD in Fig.8(a)) and $\mathcal{M}=\{1,1,2,2\}$, the PS scheme $\left[1 p_{1}, 1 p_{2}, 2 p_{3}, 2 p_{4}\right]$ generates the attractor $A^{*}$, which approximates $A_{p^{*}}$ with $p^{*}=1.38$. The match between the two attractors is revealed in Fig.8(b)-(i). Again the PS algorithm acts like a control-like method.

\section{Conclusion}

In this paper we present a new version of Chen's system: the PWL Chen system of fractional-order, revealing interesting new dynamical characteristics, including the coexistence of chaotic and regular 
motions. To numerical cope with this system, we first must make a continuous approximation of the discontinuities by using an algorithm based on Filippov's regularization and also by applying Cellina's Theorem. The approximation is implemented via the sigmoid function (7).

We show that the stable attractors can be numerically approximated with the PS algorithm by starting from a set of parameter values which are periodically switched while the underlying IVP is numerically integrated. Results are robust across a range of moderate small step sizes.

We note that the case $p:=a$ allowed us to verify that the PS algorithm applies also to a new general class of systems modeled by (9).

One of the open and most intriguing problems is revealed by following question: Even the PWL equation (3) and the continuous equation (8) are equivalent in the sense of numerical approximation, some experimental (circuitry) implementations of both system, will reveal the same behavior? A positive answer to this question will be useful to underline the importance of this approach of PWL systems.

Also, the convergence proof of the PS algorithm for other larger classes of IVPs remains the subject of future works.

Acknowledgements M.A. Aziz Alaoui is currently funded by the European Regional Development Funding via RISC project and by CPER Region Haute Normandie France.

Michal Small is currently funded by the Australian Research Council via a Future Fellowship (FT110100896) and Discovery Project (DP140100203).

\section{References}

[1] Chen G and Ueta T 1999 Int. J. Bifurc. Chaos 91465

[2] Aziz-Alaoui M A and Chen G 2002 Int. J. Bifurc. Chaos 12147

[3] Hu J-B, Zhao L-D and Xie Z-G 2013 Chin. Phys. B 22080506

[4] Liu J-G 2013 Chin. Phys. B 2260510

[5] Mainardi F 1996 Appl. Math. Lett. 923

[6] Li R-H, and Chen W-S 2013 Chin. Phys. B 2240503

[7] Kilbas A A, Srivastava H M and Trujillo J J 2006 Theory and Applications Fractional Differential Equations (North-Holland Mathematics Studies, Vol. 204, North-Holland, Amsterdam)

[8] Zhang R X, Yang Y, Yang SP and Xuebao W 2009 Acta Physica Sinica 586039

[9] Samko G, Kilbas A A and Marichev O I 1993 Fractional Integrals and Derivatives: Theory and Applications (Yverdon: Gordon and Breach)

[10] Ma T, Jiang W, Fu J, Chai Y, Chen L and Xue F 2012Acta Physica Sinica 61160506

[11] Wang S P, Lao S K, Chen H K, Chen J H and Chen S Y 2013 Int. J. Bifurc. Chaos 23 1350030

[12] Petras I 2011 Fractional-Order Nonlinear Systems: Modeling, Analysis and Simulation (Hidelberg: Springer-Verlag)

[13] Arena P, Caponetto R, Fortuna L, and Porto D 2000 Nonlinear Noninteger Order Circuits and Systems - An Introduction (Singapore: World Scientific)

[14] Wen S, Zeng Z, Huang T, and Zhang Y 2014 IEEE Transactions on Fuzzy Systems 221704

[15] Wen S, Huang T, Zeng Z, Chen Y and Lie P 2014 Neural Networks 6348

[16] Wen S P, Zeng Z G, Huang T W and Li C J 2013 International Journal of Robust and Nonlinear Control DOI: 10.1002/rnc.3112 
[17] Li C and Chen G 2004 Chaos, Solitons 6 Fractals 22549

[18] Caputo, M 1967 Geophys. J. R. Astron. Soc. 13 529; reprinted in Fract. Calc. Appl. Anal. 200710309

[19] Oldham K B and Spanier J 1974 The Fractional Calculus: Theory and Applications of Differentiation and Integration of Arbitrary Order (New York: Academic Press)

[20] Podlubny I 1999 Fractional Differential Equations (San Diego: Academic Press )

[21] Heymans N and Podlubny I 2006 Rheol. Acta 45765

[22] Diethelm K, Ford N J and Freed A D 2002 Nonlinear Dyn. 293

[23] Danca M-F and Diethlem K 2011 Comm. Nonlinear Sci. Numer Simul. 153745

[24] Danca M-F 2013 Comm. Nonlinear Sci. Numer Simul. 18500

[25] Feckan M, Danca M-F 2014 Mathematica Slovaca accepted

[26] Diethlem K 2010 The Analysis of Fractional Differential Equations (Berlin Heidelberg: Springerr-Verlag)

[27] Odibat Z, Bertelle C, Aziz-Alaoui M A and Duchamp G 2010 Comp. Math. Appl. 591462

[28] Danca M-F 2014 Int. J. Bifurc. Chaos accepted

[29] Kaslik E and Sivasundaram S 2012 Nonlinear Anal-Real 131489

[30] Falconer K 1990 Fractal Geometry, Mathematical Foundations and Applications (John Wiley and Sons: Chichester) 


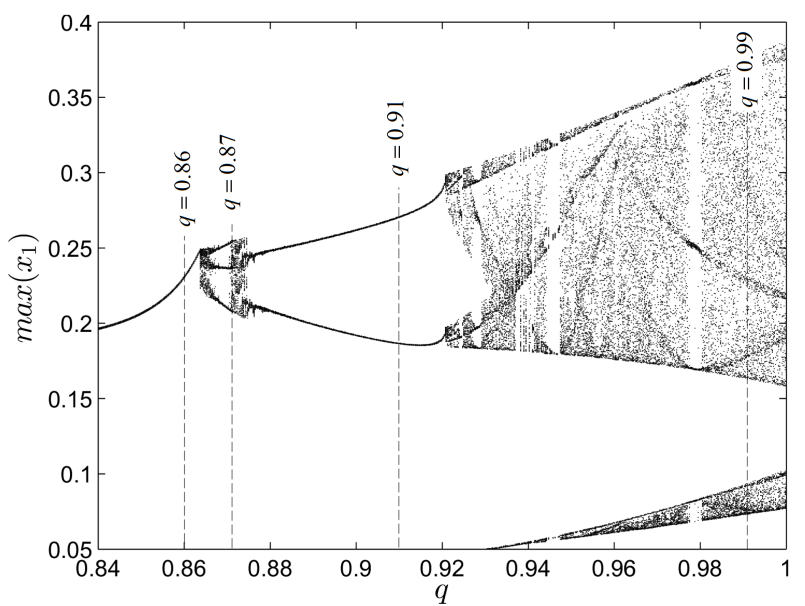

(a)

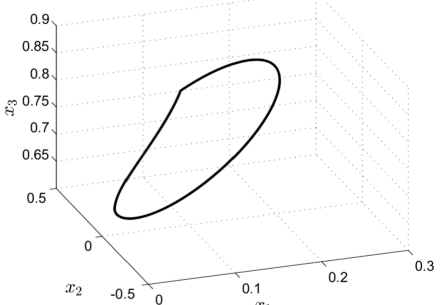

(b)

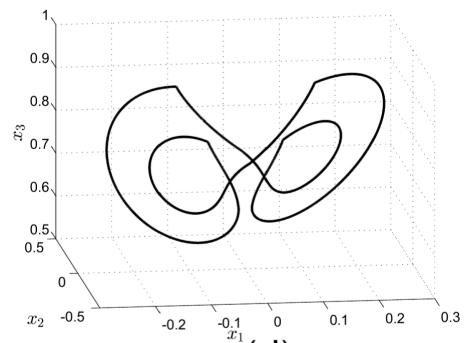

(d)

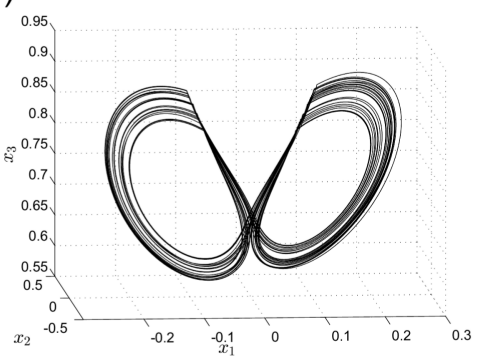

(c)

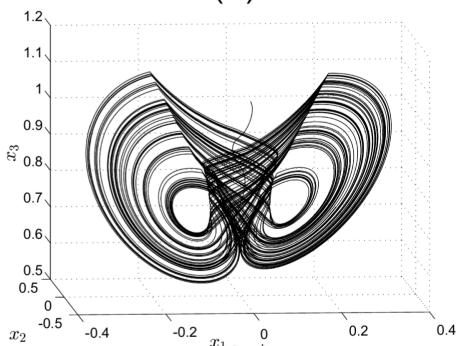

$(\mathrm{e})$

Figure 1: Bifurcation diagram of Chen's system (3) for the commensurate fractional-order $q \in$ $[0.84,1]$; (a)-(e) Attractors corresponding to $q=0.86, q=0.87, q=0.91$ and $q=0.99$ respectivelly. 

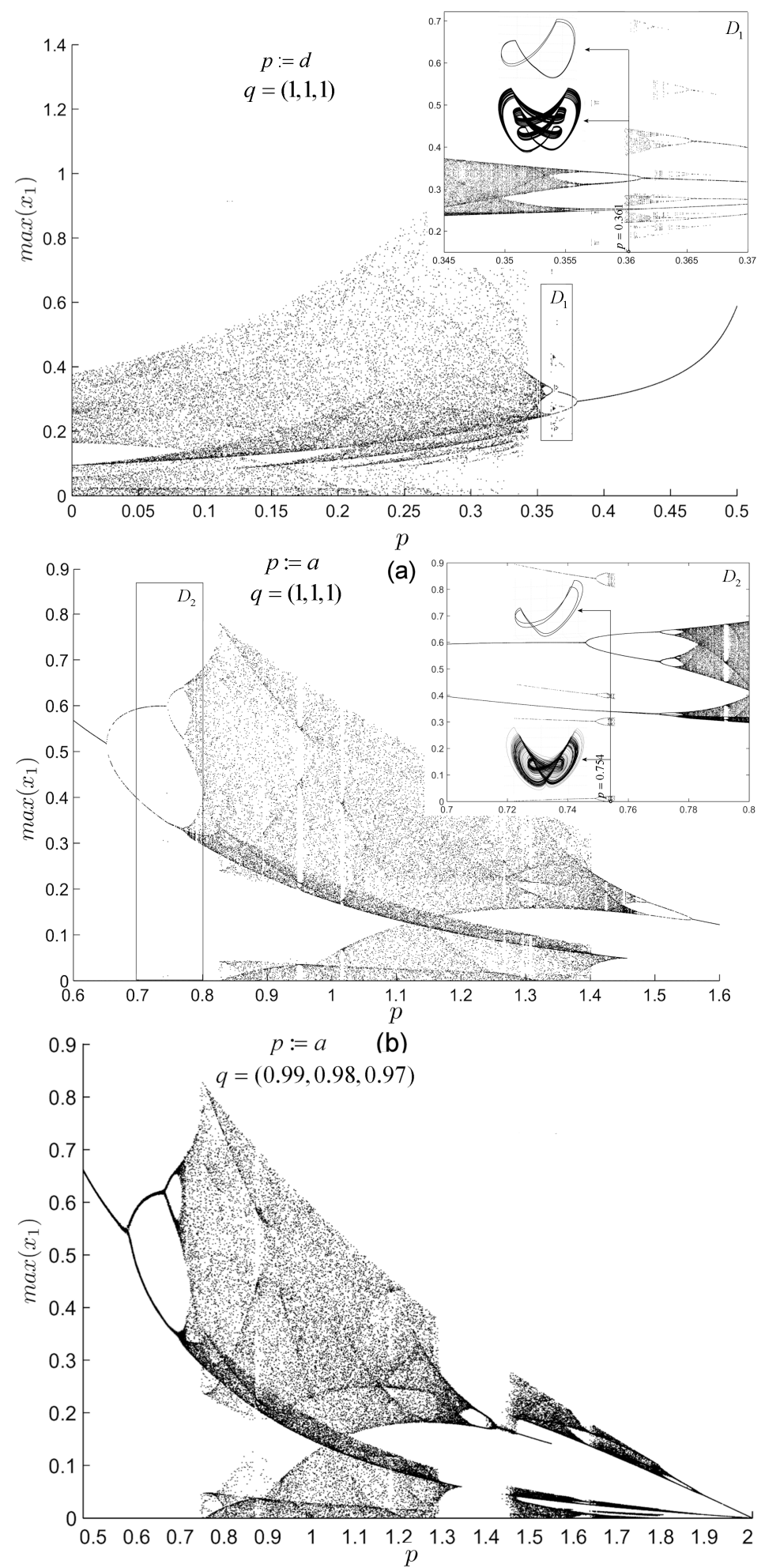

(c)

Figure 2: Bifurcation diagrams of Chen's system (3); (a) $p:=d, q=(1,1,1)$ and $a=1.15$, $c=2$ and $b=0.15$; (b) $p:=a, q=(1,1,1)$ and $b=0.15, c=2$ and $d=0.1$; (c) $p=a$, $q=(0.99,0.98,0.97)$ and $b=0.15, c=2$ and $d=0.1$. 


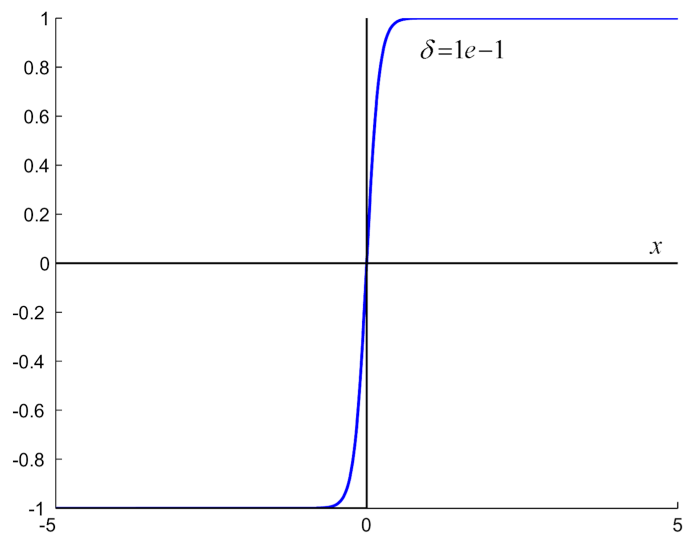

(a)

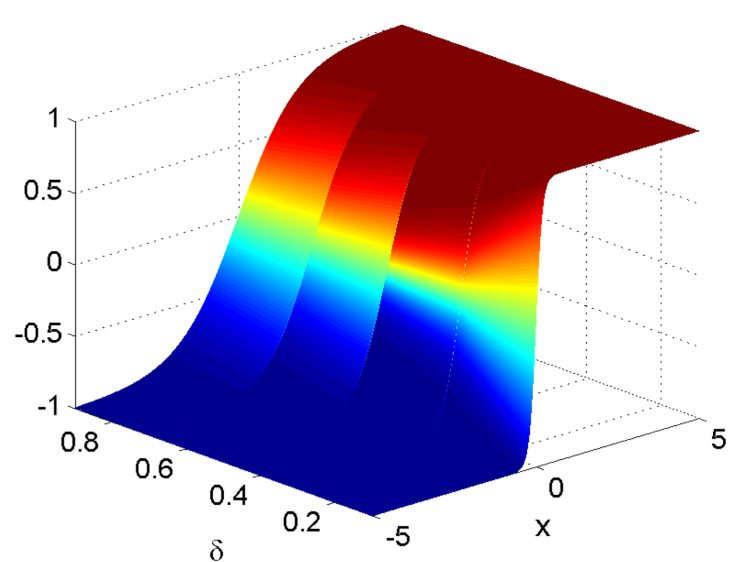

(b)

Figure 3: (a) Graph of the sigmoid function $\widetilde{\operatorname{sgn}}$ for a large value of $\delta: \delta=1 e-1$; (b) Graph of the sigmoid function depending on $\delta$. 


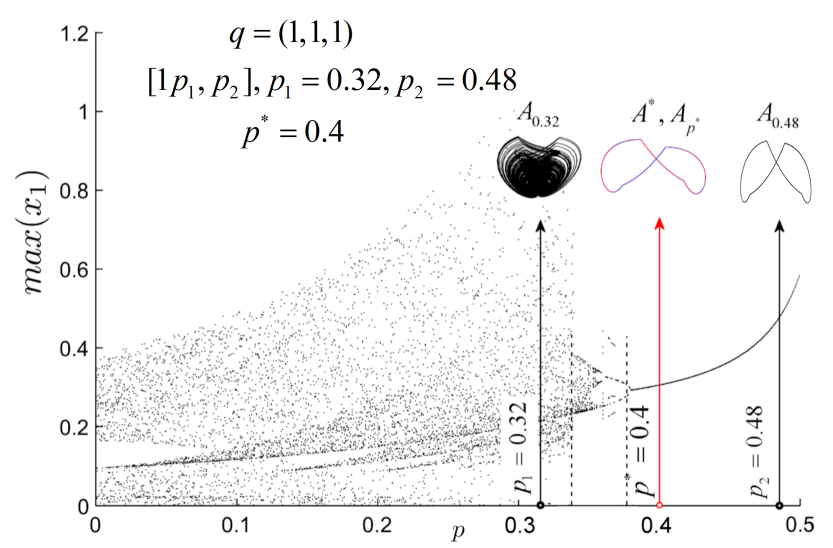

(a)

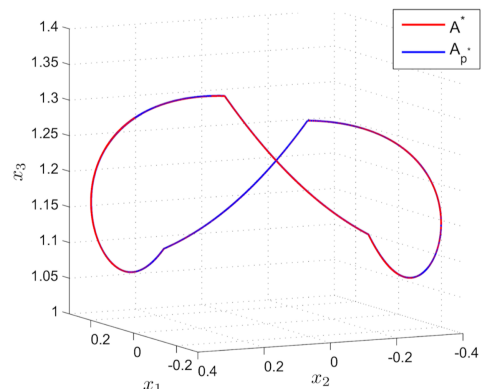

(b)

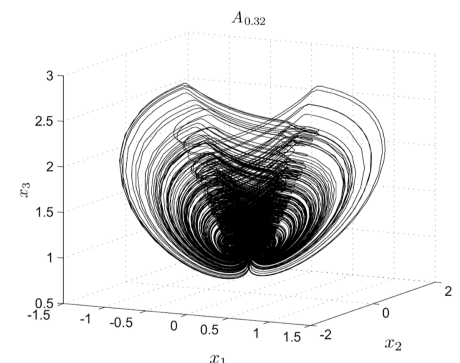

(c)

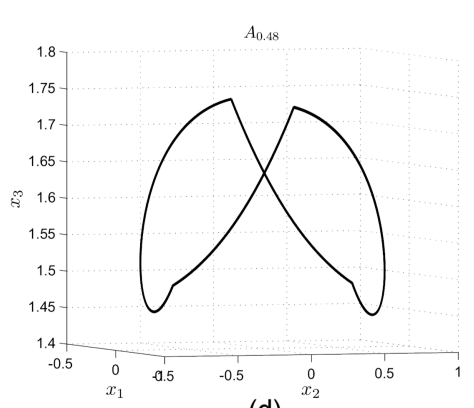

(d)

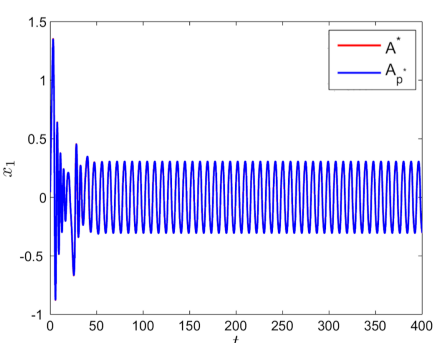

(e)

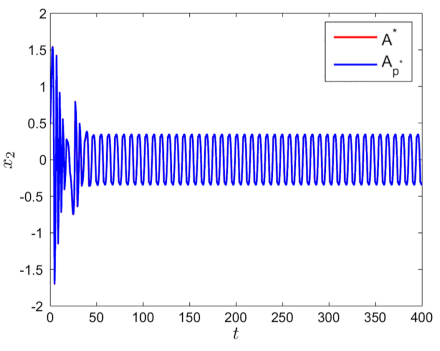

(f)

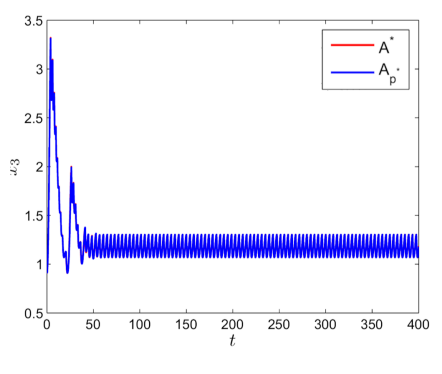

(g)

Figure 4: PWL Chen's attractor $A_{0.4}$, for $p:=d$, and $q=(1,1,1)$, approximated with PS algorithm with schme $\left[1 p_{1}, 1 p_{2}\right]$ with $p_{1}=0.32$ and $p_{2}=0.48$; (a) Bifurcation diagrams; (b) Overplotted attractors $A^{*}$ (red) and $A_{p^{*}}$ (blue); (c) Attractor $A_{0.32}$; (d) Attractor $A_{0.48}$; (e)-(g) Overplotted time series corresponding to $A^{*}$ (red) and $A_{p^{*}}$ (blue). 


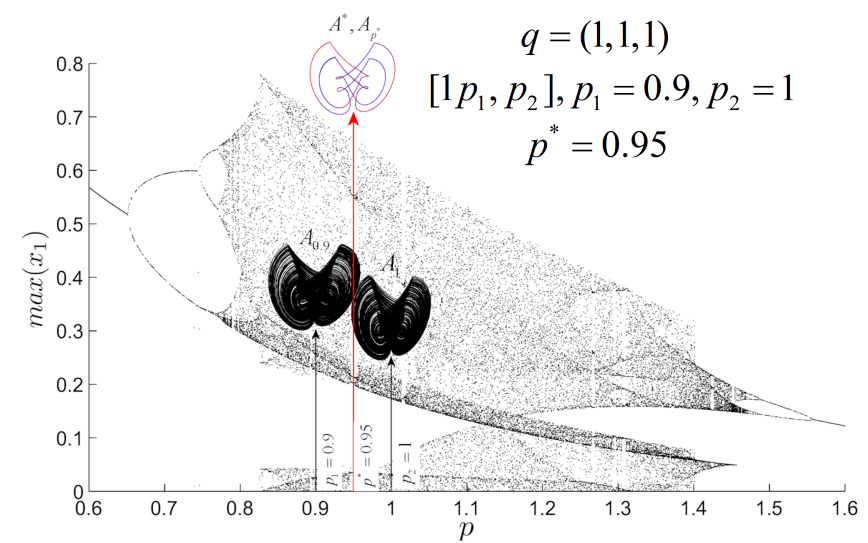

(a)

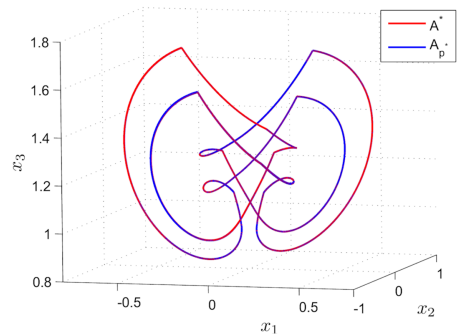

(b)

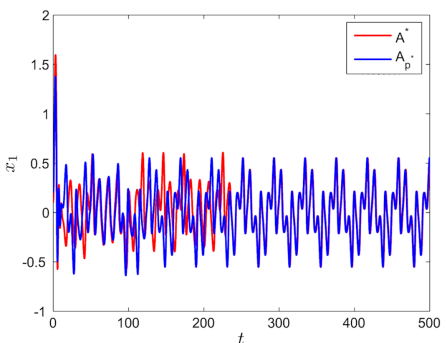

(e)
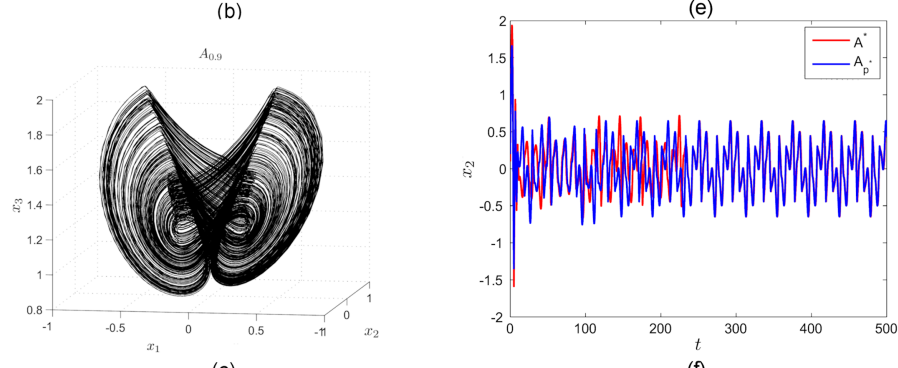

(c)

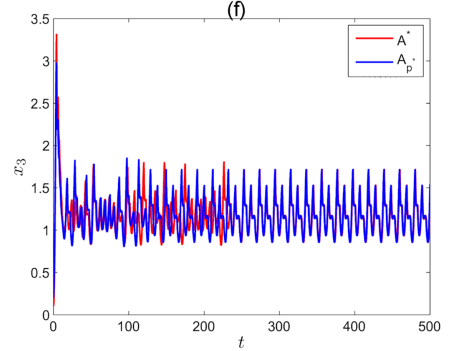

(d)

(g)

Figure 5: PWL Chen's attractor $A_{0.95}$, for $p:=a, q=(1,1,1)$, approximated with PS algorithm with scheme $\left[1 p_{1}, 1 p_{2}\right]$ with $p_{1}=0.9$ and $p_{2}=1$; (a) Bifurcation diagram; (b) Overplotted attractors $A^{*}$ (red) and $A_{p^{*}}$ (blue); (c) Attractor $A_{0.9}$; (d) Attractor $A_{1}$; (e)-(g) Overplotted time series corresponding to $A^{*}$ (red) and $A_{p^{*}}$ (blue). 


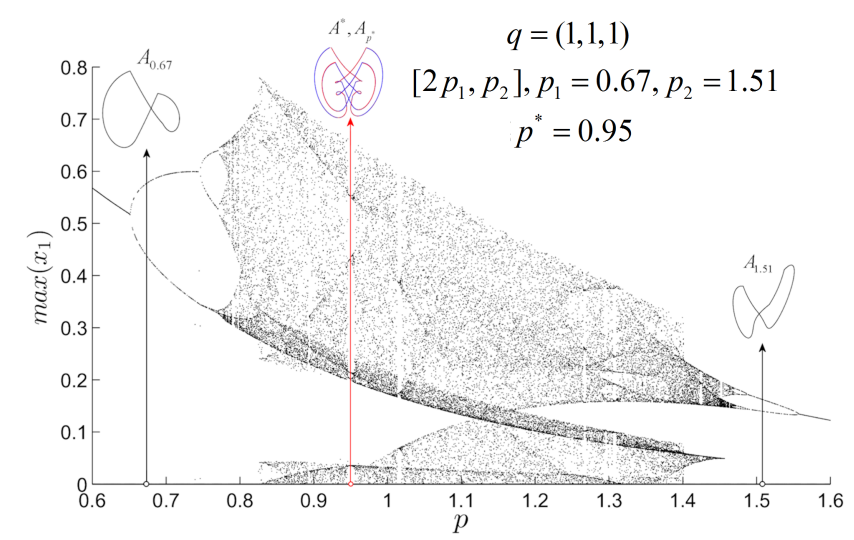

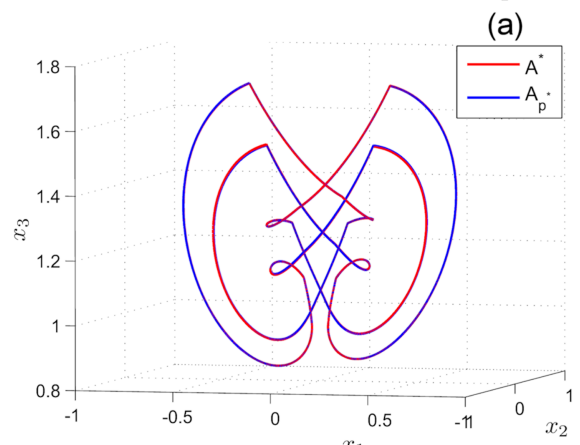

(b)

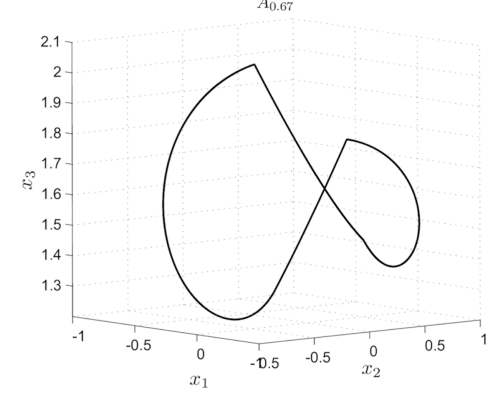

(c)

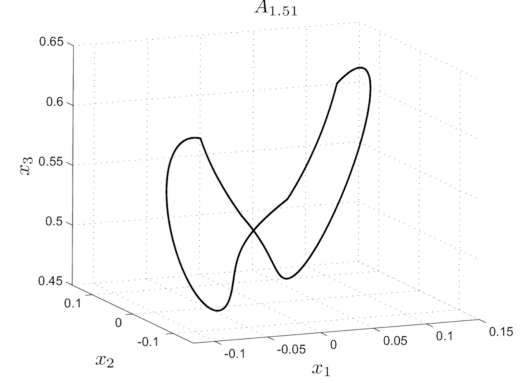

(d)

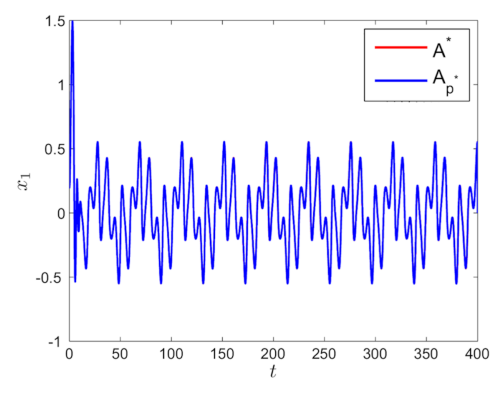

(e)

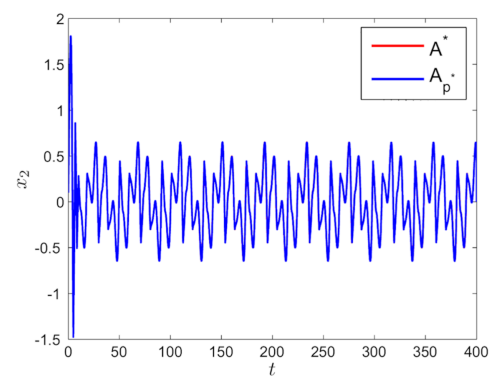

(f)

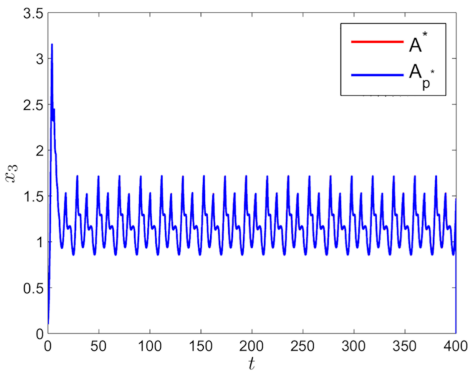

(g)

Figure 6: PWL Chen's attractor $A_{0.95}$, for $p:=a, q=(1,1,1)$, approximated with PS algorithm with scehme $\left[2 p_{1}, 1 p_{2}\right]$ with $p_{1}=0.67$ and $p_{2}=1.51$; (a) Bifurcation diagram; (b) Overplotted attractors $A^{*}$ (red) and $A_{p^{*}}$ (blue); (c) Attractor $A_{0.67}$; d) Attractor $A_{1.51}$; (e)-(g) Overplotted time series corresponding to $A^{*}$ (red) and $A_{p^{*}}$ (blue). 

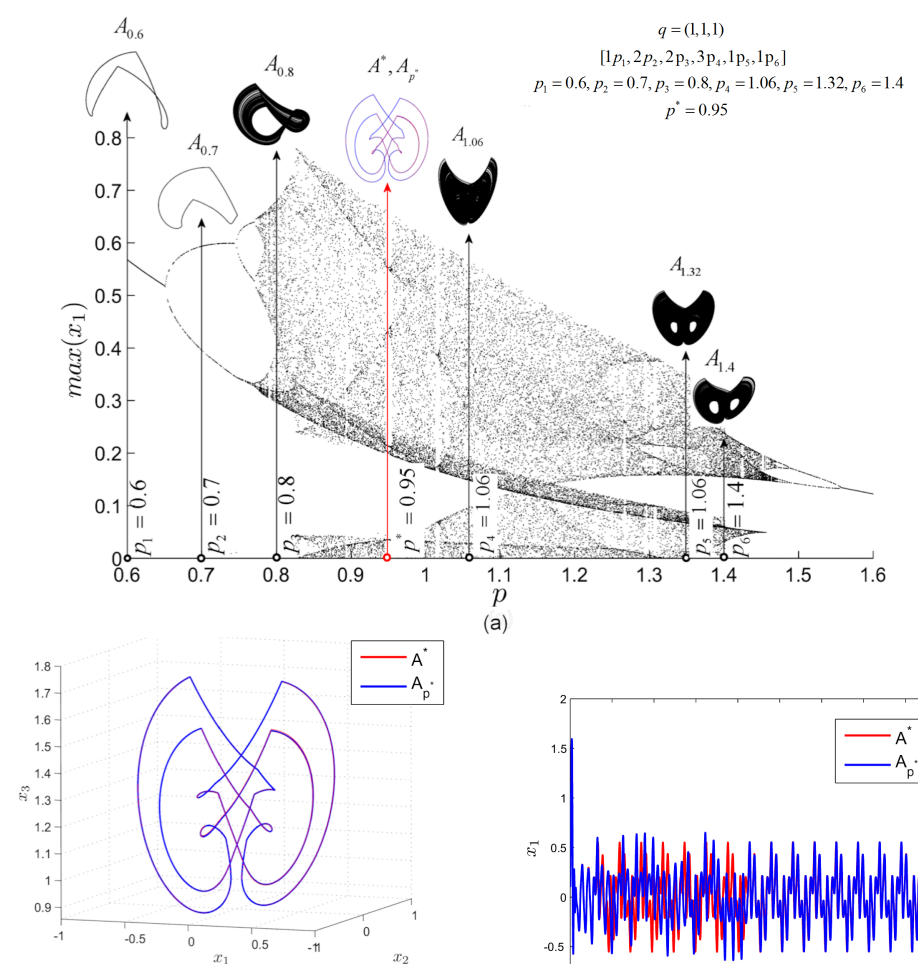

(b)

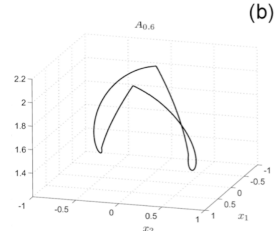

(c)

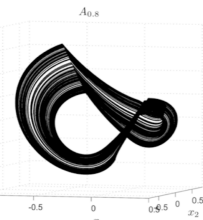

(e)

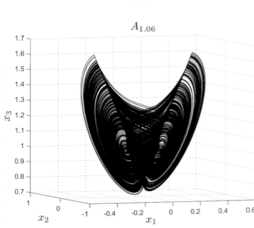

(g)

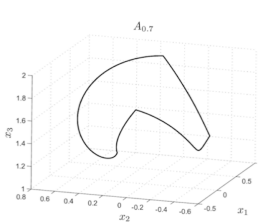

(d)

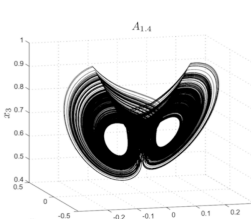

(f)

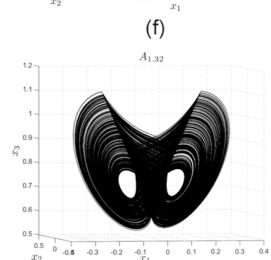

(h)

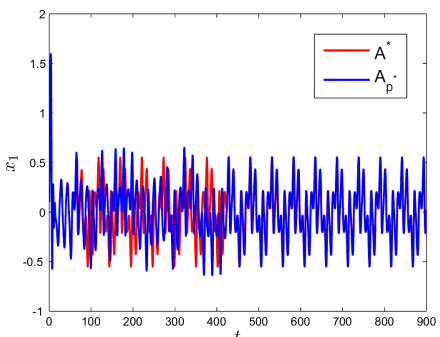

(i)
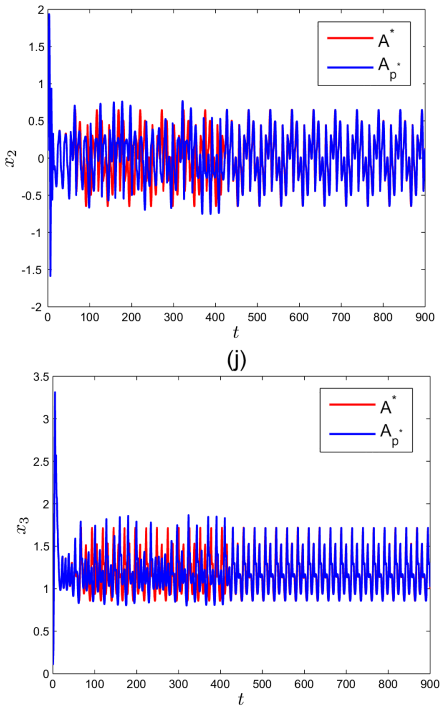

(k)

Figure 7: PWL Chen's attractor $A_{0.95}$, for $p:=a, q=(1,1,1)$, approximated with PS algorithm with the scheme $\left[1 p_{1}, 2 p_{2}, 2 p_{3}, 3 p_{4}, 1 p_{5}, 1 p_{6}\right]$ with $\mathcal{P}_{6}=\{0.6,0.7,0.8,1.09,1.32,1.4\}$; (a) Bifurcation diagram; (b) Overplotted attractors $A^{*}$ (red) and $A_{p^{*}}$ (blue); (c)-(h) Attractors $A_{0.6}, A_{0.7}, A_{0.8}$, $A_{1.09}, A_{1.32}$ and $\left.A_{1.4} ; \mathrm{i}-\mathrm{k}\right)$ Overplotted time series corresponding to $A^{*}$ (red) and $A_{p^{*}}$ (blue) 


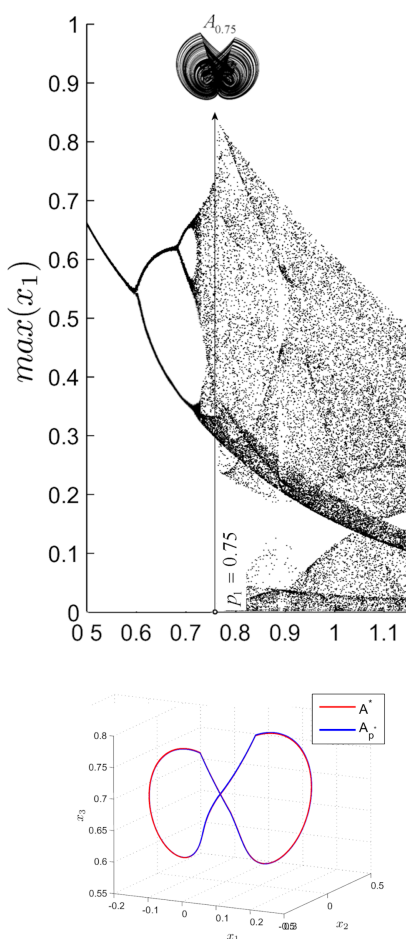

(b)

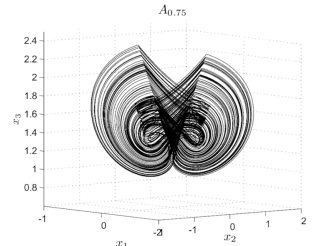

(c)

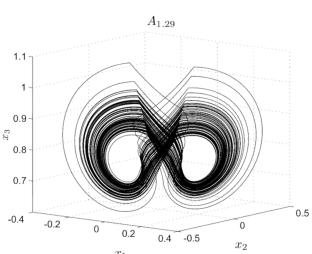

(d)

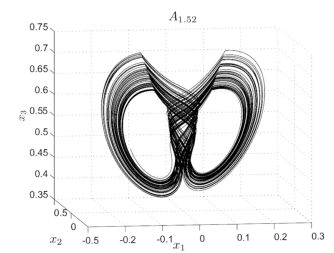

(e)

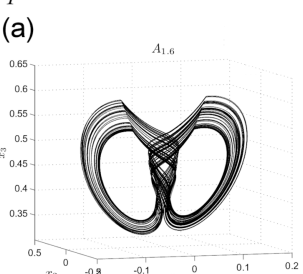

(f)

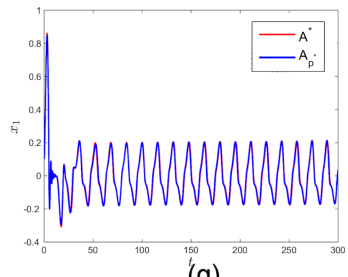

$(\mathrm{g})$

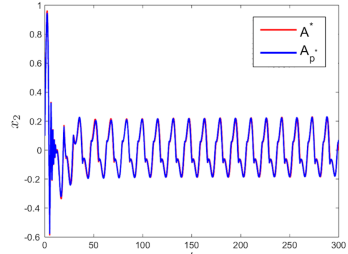

(h)

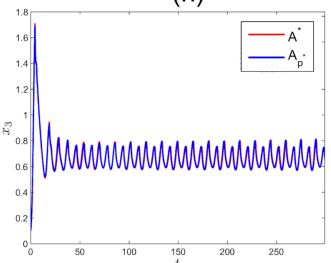

(i)

Figure 8: PWL Chen's attractor $A_{1.38}$, for $p:=a, q=(0.99,0.98,0.97)$, approximated with PS algorithm with the scheme $\left[1 p_{1}, 1 p_{2}, 2 p_{3}, 2 p_{4}\right]$ with $\mathcal{P}_{4}=\{0.75,1.29,1.52,1.6\}$; (a) Bifurcation diagram; (b) Overplotted attractors $A^{*}$ (red) and $A_{p^{*}}$ (blue); (c)-(g) Attractors $A_{0.75}, A_{1.29}, A_{1.52}$, $A_{1.6}$ and $A_{1.38}$; (h)-(j) Overplotted time series corresponding to $A^{*}$ (red) and $A_{p^{*}}$ (blue). 